\title{
Chandra X-Ray Observations of the Redshift 1.53 Radio-Loud Quasar 3c 270.1
}

\section{Citation}

Wilkes, Belinda J., Dharam V. Lal, D. M. Worrall, Mark Birkinshaw, Martin Haas, S. P. Willner, Robert Antonucci, et al. 2012. "Chandra X-Ray Observations of the Redshift 1.53 Radio-Loud Quasar 3c 270.1." The Astrophysical Journal 745 (1) (January 3): 84. doi:10.1088/0004-637x/745/1/84.

\section{Published Version}

doi:10.1088/0004-637X/745/1/84

\section{Permanent link}

http://nrs.harvard.edu/urn-3:HUL.InstRepos:29921926

\section{Terms of Use}

This article was downloaded from Harvard University's DASH repository, and is made available under the terms and conditions applicable to Other Posted Material, as set forth at http:// nrs.harvard.edu/urn-3:HUL.InstRepos:dash.current.terms-of-use\#LAA

\section{Share Your Story}

The Harvard community has made this article openly available.

Please share how this access benefits you. Submit a story.

\section{Accessibility}




\title{
CHANDRA X-RAY OBSERVATIONS OF THE REDSHIFT 1.53 RADIO-LOUD QUASAR 3C 270.1
}

\author{
Belinda J. Wilkes ${ }^{1}$, Dharam V. LaL ${ }^{1}$, D. M. Worrall ${ }^{2}$, Mark Birkinshaw ${ }^{2}$, Martin HaAs ${ }^{3}$, S. P. Willner ${ }^{1}$, \\ Robert Antonucci ${ }^{4}$, M. L. N. Ashby ${ }^{1}$, Mark Avara ${ }^{5}$, Peter Barthel ${ }^{6}$, Rolf Chini $^{3,7}$, G. G. Fazio ${ }^{1}$, Martin Hardcastle ${ }^{8}$, \\ Charles Lawrence ${ }^{9}$, Christian Leipski ${ }^{10}$, Patrick Ogle ${ }^{11}$, and Bernhard Schulz ${ }^{12}$ \\ ${ }^{1}$ Harvard-Smithsonian Center for Astrophysics, Cambridge, MA 02138, USA \\ ${ }^{2}$ HH Wills Physics Laboratory, University of Bristol, Bristol, UK \\ ${ }_{3}^{3}$ Astronomisches Institut, Ruhr-University, Bochum, Germany \\ ${ }^{4}$ Department of Physics, University of California, Santa Barbara, CA 93106, USA \\ ${ }^{5}$ Department of Astronomy, University of Maryland, College Park, MD 20742-2421, USA \\ ${ }^{6}$ Kapteyn Astronomical Institute, University of Groningen, Groningen, The Netherlands \\ ${ }^{7}$ Instituto de Astronomía, Universidad Católica del Norte, Antofagasta, Chile \\ ${ }^{8}$ School of Physics and Astronomy, University of Hertfordshire, Hatfield, UK \\ ${ }^{9}$ JPL, Pasadena, CA 91109, USA \\ ${ }^{10}$ MPIA, Heidelberg, Germany \\ ${ }^{11}$ Spitzer Science Center, Caltech, Pasadena, CA 91125, USA \\ 12 IPAC, Caltech, Pasadena, CA 91125, USA \\ Received 2011 June 15; accepted 2011 September 8; published 2012 January 3
}

\begin{abstract}
Chandra X-ray observations of the high redshift $(z=1.532)$ radio-loud quasar 3C 270.1 in 2008 February show the nucleus to have a power-law spectrum, $\Gamma=1.66 \pm 0.08$, typical of a radio-loud quasar, and a marginally detected $\mathrm{Fe} \mathrm{K} \alpha$ emission line. The data also reveal extended X-ray emission, about half of which is associated with the radio emission from this source. The southern emission is co-spatial with the radio lobe and peaks at the position of the double radio hot spot. Modeling this hot spot, including Spitzer upper limits, rules out synchrotron emission from a single power-law population of electrons, favoring inverse Compton emission with a field of $\sim 11 \mathrm{nT}$, roughly a third of the equipartition value. The northern emission is concentrated close to the location of a $40^{\circ}$ bend where the radio jet is presumed to encounter an external medium. It can be explained by inverse Compton emission involving cosmic microwave background photons with a field of $\sim 3 \mathrm{nT}$, a factor of 7-10 below the equipartition value. The remaining, more diffuse $\mathrm{X}$-ray emission is harder $(\mathrm{HR}=-0.09 \pm 0.22)$. With only $22.8 \pm 5.6$ counts, the spectral form cannot be constrained. Assuming thermal emission with a temperature of $4 \mathrm{keV}$ yields an estimate for the luminosity of $1.8 \times 10^{44} \mathrm{erg} \mathrm{s}^{-1}$, consistent with the luminosity-temperature relation of lower-redshift clusters. However, deeper Chandra X-ray observations are required to delineate the spatial distribution and better constrain the spectrum of the diffuse emission to verify that we have detected X-ray emission from a high-redshift cluster.
\end{abstract}

Key words: quasars: individual: 3C 270.1 - X-rays: galaxies: clusters

Online-only material: color figures

\section{INTRODUCTION}

With the aim of quantifying the orientation dependence of the observed properties of quasars, we have embarked on multiwavelength observations of high-redshift $(1 \leqslant z \leqslant 2)$ radio sources with Chandra, Spitzer, Herschel, and ground-based observatories. Given the known orientation dependence of the emission from quasars and active galactic nuclei (AGNs) and the resulting strong selection bias against obscured/edge-on sources, isotropic, low-frequency radio emission provides a rare, unbiased view of the population based on optically thin emission far from the nucleus (i.e., lobe selection). We chose high-redshift $3 \mathrm{CR}$ (selected at $178 \mathrm{MHz}$ ) sources to ensure that the sample is largely unbiased and that it comprises powerful radio galaxies and quasars. Studies of high-redshift, radio-loud quasars also facilitate more lines of investigation: X-ray emission is related to radio structure at high redshift, and thus the interaction of the quasar with its environment, and searches for high-redshift clusters of galaxies.

$\mathrm{X}$-ray emission is often observed from radio-emitting hot spots and lobes in quasars. It is generally interpreted (Harris \& Krawczynski 2002; Worrall 2009) in terms of direct synchrotron emission from high-energy electrons, inverse Compton (iC) emission due to upscattering of radio photons within the radio hot spots (synchrotron self-Compton, SSC; Hardcastle et al. 2004), or iC upscattering of external radio photons in the extended lobes, most likely from the cosmic microwave background (iC/CMB; Croston et al. 2005). The latter will be more luminous for larger radio-emitting structures and at higher redshift due to the higher energy density of the CMB.

Luminous high-redshift radio sources occur in massive galaxies and so, according to the hierarchical paradigm, form at peaks in the dark matter density. Thus, they are beacons for high-density regions in the early universe and for high-redshift clusters and groups, very few of which are yet known. Finding clusters at these high redshifts is key to the study of both cluster and galaxy formation and will provide critical constraints on theoretical models for cluster and galaxy evolution (Andreon et al. 2011; Hilton et al. 2010; Venemans et al. 2007) and the mass distribution of dark matter halos (Croton et al. 2007).

The radio-loud quasar 3C $270.1(z=1.532)$ has the doublelobed radio structure characteristic of FR-II (Fanaroff \& Riley 1974) radio sources and the strong, broad emission lines of a type 1 quasar. Optical and infrared (IR) data show an excess of galaxies within $\sim 640 \mathrm{kpc}\left(1^{\prime} \cdot 3\right)$ of the quasar suggesting a surrounding cluster of galaxies (Haas et al. 2009). The sky 

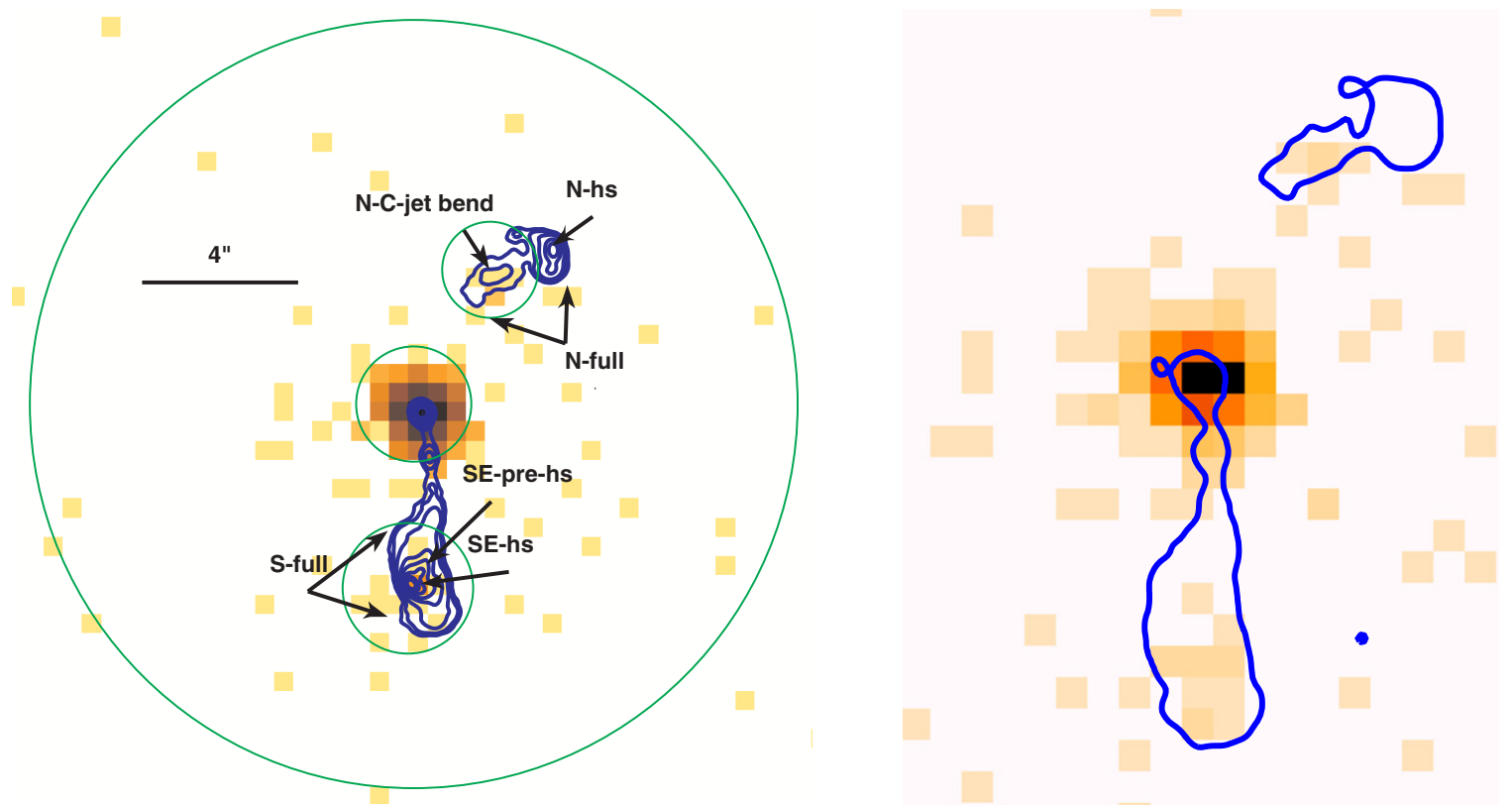

Figure 1. Left: radio contours in blue derived from the VLA radio map at $8.46 \mathrm{GHz}$ ( $X$-band) superposed on the unsmoothed, broadband (0.3-8 keV) Chandra X-ray data at native (0!'492) pixelation. North is up and east is to the left. Radio contour levels are: $0.2,0.36,0.96,5.12,12.8,25.6,38.4,54.4$, and 76.8 mJy beam ${ }^{-1}$. The scale bar indicates $4^{\prime \prime}(34 \mathrm{kpc})$ and the outer circle has radius $10^{\prime \prime}$. Key morphological features discussed in the text are labeled. The X-ray extraction regions for the quasar, the radio-related emission north and south of 3C 270.1, and the inner edge of the background region annulus is shown in green. Right: zoom in on the central regions including the lowest radio contour level to highlight the radio-X-ray alignment.

distribution of the cluster galaxy candidates forms a loose concentration centered $\sim 20^{\prime \prime}$ east of the quasar, although the centroid is not well constrained by the current data.

This paper reports Chandra observations of the X-ray emission from the vicinity of $3 \mathrm{C} 270.1$. This includes unresolved emission from the quasar itself and extended X-ray emission within $\sim 10^{\prime \prime}$ of the core. The extended X-rays have two components: softer emission associated with the radio emission north and south of the core and harder, more diffuse emission which may originate in gas associated with the cluster in which the quasar is embedded (Haas et al. 2009) or in iC/CMB emission from low-surface-brightness or older radio emission that is not easily detectable (Fabian et al. 2003).

We assume a $\Lambda$ CDM cosmology with $H_{o}=71 \mathrm{~km} \mathrm{~s}^{-1} \mathrm{Mpc}^{-1}$, $\Omega_{M}=0.27$, and $\Omega_{\text {vac }}=0.73$ (Larson et al. 2011).

\section{MULTI-WAVELENGTH OBSERVATIONS AND DATA}

3C 270.1 was observed on-axis with the Chandra ACIS-S for $9.673 \mathrm{ks}$ on 2008 February 16 (ObsID 9255). The data were reprocessed in 2011 January with CIAO 4.3 to take advantage of the latest calibration files and CTI (charge transfer inefficiency) calibration as well as sub-pixel event repositioning, which improves the spatial resolution. The reprocessed data were used in the analysis presented here. The quasar is well detected with $>700$ counts consistent with the spatial distribution of a point source. There are also $>40$ excess counts within $10^{\prime \prime}$ of the quasar but outside its point-spread function (PSF).

Radio data from the Very Large Array (VLA) archive were reprocessed to generate high-resolution images at 1.43, 8.46, and $14.94 \mathrm{GHz}$ to provide comparison images at resolution similar to, or better than, that of the Chandra data. All processing was done in AIPS and followed the usual steps of calibration, imaging, and a CLEANing/self-calibration cycle. The data sets used are listed in Table 1, with the angular sizes of the
Table 1

VLA Radio Data

\begin{tabular}{llcl}
\hline \hline Project & Date Observed & $\begin{array}{c}\text { Frequency } \\
(\mathrm{GHz})\end{array}$ & $\begin{array}{c}\text { Full-resolution Gaussian } \\
\text { Beam FWHM } \\
\text { major } \times \text { minor }(\text { P.A. }) \\
\left(\operatorname{arcsec}^{2}\right)(\mathrm{deg})\end{array}$ \\
\hline AB796 & 1996 Nov 7 & 8.46 & $0.25 \times 0.24\left(120^{\circ} 5\right)$ \\
AH480 & 1992 Dec 5 & 1.43 & $1.30 \times 1.18\left(35^{\circ} 9\right)$ \\
AL073 & 1983 Oct 21 & 14.94 & $0.13 \times 0.12\left(69^{\circ} 4\right)$ \\
\hline
\end{tabular}

synthesized beams generated from the full data sets at uniform sampling.

Spitzer/IRAC data for 3C 270.1 were obtained on 2007 June 28 and are reported by Haas et al. $(2008,2009)$. The on-source exposure times of $4 \times 30 \mathrm{~s}$ in each band allow us to detect point sources to $\sim 3 \mu \mathrm{Jy}(3 \sigma)$ at 3.6 and $4.5 \mu \mathrm{m}$ and $\sim 25 \mu \mathrm{Jy}$ at 5.8 and $8 \mu \mathrm{m}$.

We obtained $z^{\prime}\left(\lambda_{\text {eff }} \sim 9049 \AA\right.$ A Fukugita et al. 1996) imaging of 3C 270.1 in 2007 June with Megacam (McLeod et al. 2006) at the $6.5 \mathrm{~m}$ MMT. The total integration time was 40 minutes under conditions of subarcsecond seeing. The individual exposures were reduced using standard techniques to create a final flattened and flux-calibrated mosaic covering 3C 270.1.

\section{DATA ANALYSIS}

\subsection{Radio Morphology and Analysis}

An $8.46 \mathrm{GHz}$ image of 3C 270.1 (contours displayed in Figure 1) shows strong, unresolved emission from the central quasar and more diffuse emission from the lobes north and south of the nucleus with some extension back toward the quasar nucleus. The peaks of emission in the northern hot spot, the core, and the southern hot spot are not co-linear.

The southern emission includes a jet with position angle (P.A.) close to $180^{\circ}$ and an extensive lobe at the same P.A. 
Table 2

Summary of Radio Extraction Regions and Flux Densities and Spitzer Upper Limits Used in the SSC and iC/CMB Modeling

\begin{tabular}{|c|c|c|c|c|c|c|c|c|}
\hline Region & $\begin{array}{l}\text { R.A. } \\
\text { J2000 }\end{array}$ & $\begin{array}{c}\text { Decl. } \\
\text { J2000 }\end{array}$ & $\begin{array}{c}\text { Dimensions }^{\mathrm{a}} \\
\text { of Extraction } \\
\text { Region }\end{array}$ & Angle $^{b}$ & $\begin{array}{c}S_{1.43} \\
(\mathrm{mJy})\end{array}$ & $\begin{array}{c}S_{8.46} \\
(\mathrm{mJy})\end{array}$ & $\begin{array}{l}S_{14.93} \\
\text { (mJy) }\end{array}$ & $\begin{array}{c}\text { Spitzer } \\
\text { Upper Limits }^{\mathrm{c}}(\mu \mathrm{Jy}) \\
3.6,4.5,5.8,8.0 \mu \mathrm{m}\end{array}$ \\
\hline N-hs & $12: 20: 33.61$ & $+33: 43: 16.1$ & $00^{\prime \prime} 16 \times 00^{\prime \prime} 09$ & $00^{\circ}$ & & & 20 & \\
\hline $\mathrm{N}-\mathrm{C}$-jet-bend & $12: 20: 33.73$ & $+33: 43: 15.1$ & $1 . .0 \times 0.7$ & $30^{\circ}$ & 60 & 7 & & $2.1,3.6,23,27$ \\
\hline $\mathrm{N}$-full & $12: 20: 33.65$ & $+33: 43: 15.6$ & $1^{\prime \prime} 05 \times 1^{\prime \prime} .05$ & $00^{\circ}$ & 570 & 65 & & \\
\hline SE-hs & $12: 20: 33.90$ & $+33: 43: 07.5$ & $0.27 \times 00^{\prime \prime} 10$ & $120^{\circ}$ & & 135 & 85 & 2.1,3.6,23,27 \\
\hline SE-pre-hs & $12: 20: 33.88$ & $+33: 43: 07.9$ & $0^{\prime \prime} 22 \times 0^{\prime \prime} 16$ & $00^{\circ}$ & & & 10 & \\
\hline S-full ${ }^{\mathrm{d}}$ & $12: 20: 33.87$ & $+33: 43: 07.8$ & $2^{\prime \prime} .1 \times 0^{\prime \prime} 8$ & $00^{\circ}$ & 1630 & 230 & & $7.8,11,49,72$ \\
\hline
\end{tabular}

Notes.

a Semimajor $\times$ semiminor axes of an ellipse, with volume determined as a uniformly filled, prolate ellipsoid, except for S-full where the

dimensions are the height and radius of a right circular cylinder.

$\mathrm{b}$ The angle of rotation of the major axis to the east of north.

c $3.6 \sigma$ upper limits for a point/extended source as appropriate.

d Radio flux for the full lobe with the hot spot emission subtracted.

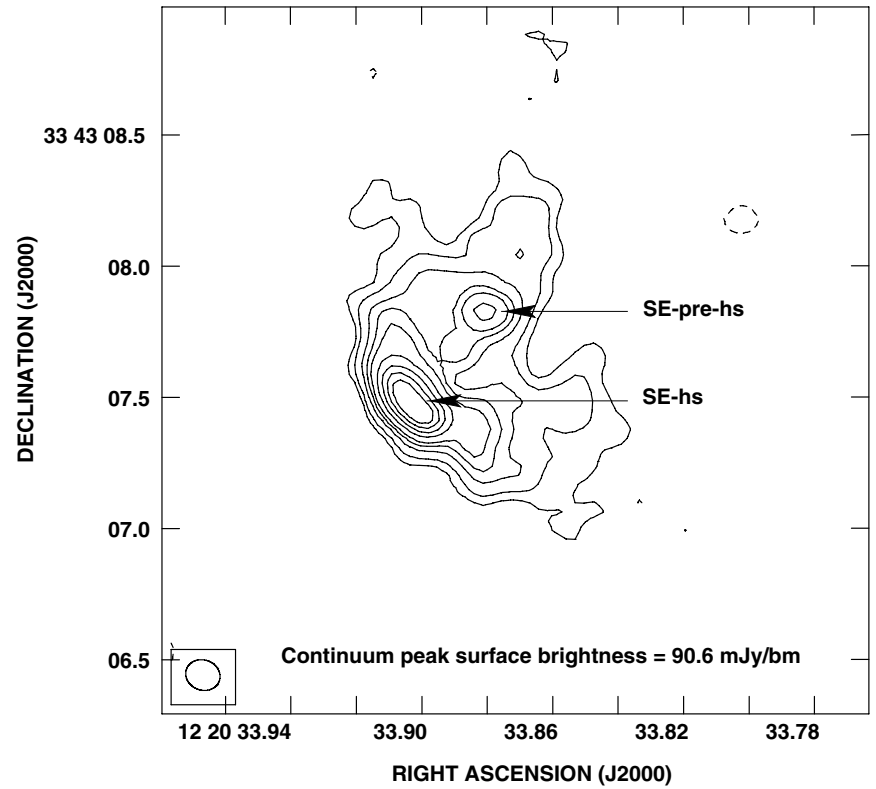

Figure 2. Radio contour plot of the $14.9 \mathrm{GHz}$ ( $U$-band) VLA data for the southern hot spot showing the morphology of the southeast (SE) and precursor hot spots as described in the text. Contour levels are: $3 \times 0.22 \times$ $(-1,1,2,4,6,8,12,16,20,24,32) \mathrm{mJy} \mathrm{bm}^{-1}$, and the beam is shown in the lower left corner.

extending over $\sim 4^{\prime \prime}(\sim 34 \mathrm{kpc})$ which includes a hot spot at the southeast (SE) edge. The jet includes a bright knot just south of the quasar core. There is no apparent excess of $\mathrm{X}$-ray counts at this position, although the resolution of the $\mathrm{X}$-ray data is sufficiently low that a deconvolution would be challenging. Figure 2 shows higher-resolution $14.9 \mathrm{GHz}$ radio contours of the southern hot spot region. There is a double radio hot spot: "precursor" and "southeast (SE)" components, with a pronounced zig-zag morphology similar to 3C 205 (Lonsdale \& Barthel 1984). A ridge of emission starts at the location of the precursor and extends toward the peak of the SE hot spot at a P.A. of $\sim 140^{\circ}$. The ridge is brighter close to the precursor hot spot in the $15 \mathrm{GHz}$ image (Figure 2) and becomes fainter farther away from it. From the peak of the SE hot spot, the ridge line turns sharply west, curving through $\sim 90^{\circ}$ to a secondary peak and decreasing in surface brightness. The length of this curving ridge is about $1^{\prime \prime}(\sim 9 \mathrm{kpc})$.
The northern lobe is skewed by $30^{\circ}$ from the axis defined by the southern jet and consists of two distinct components (Lonsdale et al. 1993). The well-defined lobe emission includes a hot spot at the end farthest from the quasar. The hot spot lies in an emission ridge with north-south orientation and is approximately 0 .'3 $(2.6 \mathrm{kpc})$ in length. A faint radio emission component extends SE from the lobe with a P.A. of $115^{\circ}$ and is a relatively diffuse, steep-spectrum feature with a length of $\sim 2^{\prime \prime} .2(\sim 19 \mathrm{kpc})$. The feature turns through an angle of $\sim 40^{\circ}$ back toward the quasar, suggesting an encounter between an outflow of radio plasma and a dense medium. We shall call this region the counter-jet-bend (N-C-jet-bend). There is no jet emission visible on the north side, and the lobe emission is significantly more depolarized (Garrington et al. 1991), further supporting the presence of hot gas surrounding the quasar and consistent with this being the counter-jet side.

We used VLA $X$-band $(8.46 \mathrm{GHz})$ and $U$-band $(14.9 \mathrm{GHz})$ radio data (Figures 1 and 2 ) to estimate radio-emitting volumes, flux densities, and spectral slopes in six regions defined based on the radio morphology: N-hs (north hot spot), SE-hs (southeast hot spot), SE-pre-hs (southeast precursor hot spot), N-C-jetbend (north counter-jet-bend), N-full (north lobe), and S-full (south lobe; Table 2). These regions are also used as the basis for determining the optical, IR, and X-ray fluxes/limits, although the spatial resolution is lower in the latter two bands so that hot-spot- or lobe-related emission cannot be separated.

\subsection{X-Ray Data Analysis}

The unsmoothed, broadband, X-ray data are shown in Figure 1 with the radio contours superposed. The X-ray spatial resolution is $\sim 0.5$, about twice that of the radio data. The X-ray data were shifted 0'.32 to the SW (Chandra's positional offset has 0.'4 rms radius, Proposers' Observatory Guide) to align the peak of the nuclear emission with the VLA position of the radio core.

The analysis of the data from several regions in the vicinity of 3C 270.1 is described below along with that of a lower-redshift $(z=1.038)$ quasar NGC 4395 B06, 48" north of 3C 270.1 (on the same ACIS-S chip and with similar PSF), which is used as a comparison point source. Extracted counts and derived fluxes are listed in Table 3.

\subsubsection{The Quasar Core}

The quasar core is unresolved and well detected. Counts were extracted from a $1^{\prime \prime} .5$ radius, circular region centered on the 

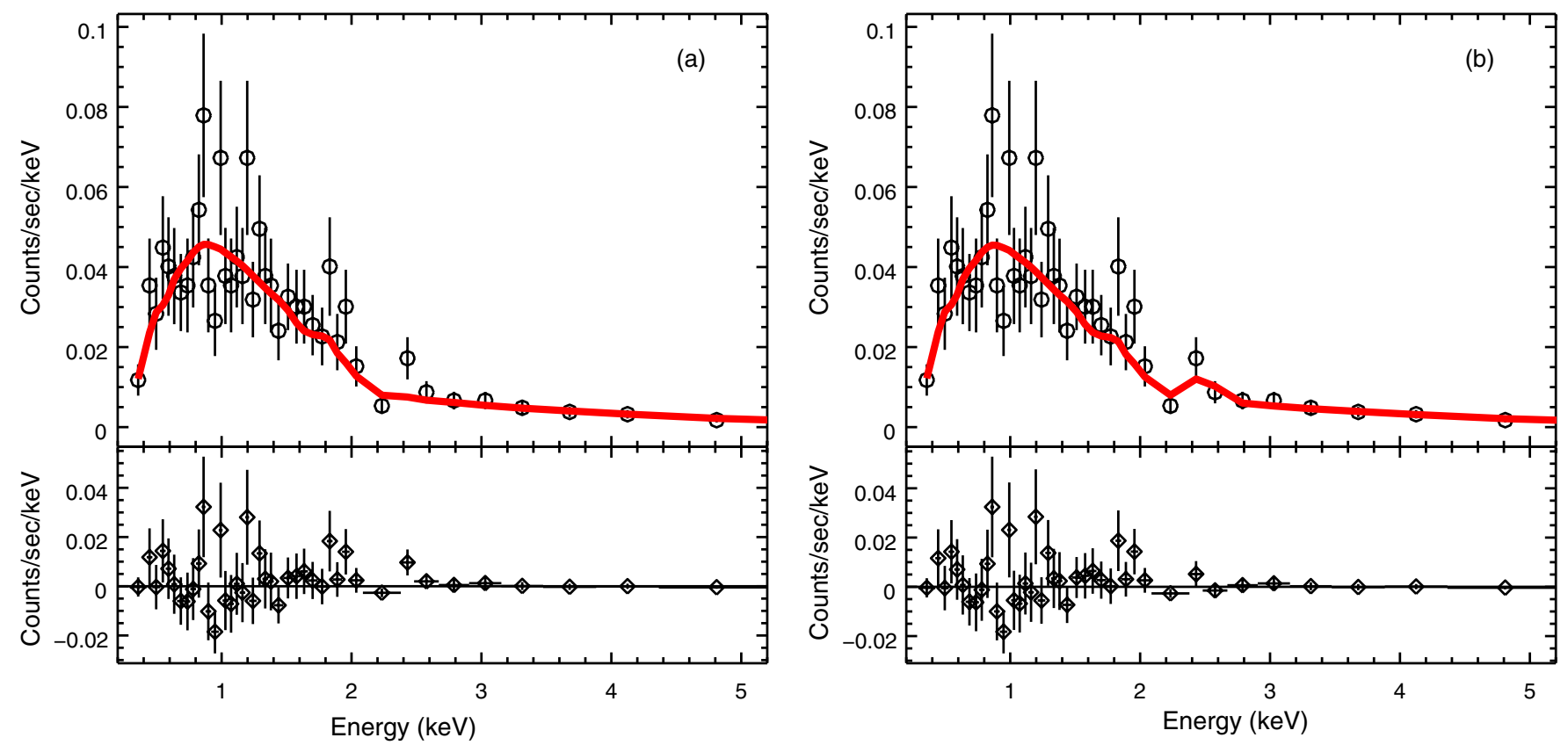

Figure 3. (a) Chandra X-ray spectrum of $3 \mathrm{C} 270.1$ with the best-fit power-law model ( $\Gamma=1.64 \pm 0.08$, Galactic $\left.N_{\mathrm{H}}\right)$ superposed and the residuals below. Deviations in the residuals at $\sim 2.5 \mathrm{keV}$ suggest the presence of an emission line. (b) As for (a), but including an Fe K $\alpha$ emission line (EW=172 $\pm 110 \mathrm{eV}$ ) fixed at energy $2.5 \mathrm{keV}$, consistent with $\mathrm{Fe} \mathrm{K} \alpha$ from cold material at the redshift of the quasar.

(A color version of this figure is available in the online journal.)

Table 3

X-Ray Counts and Derived X-Ray Parameters ${ }^{\mathrm{a}}$

\begin{tabular}{|c|c|c|c|c|c|c|}
\hline \multirow[t]{2}{*}{ Region Name } & \multirow{2}{*}{$\begin{array}{l}\text { Net Counts } \\
0.3-8 \mathrm{keV}\end{array}$} & \multirow[t]{2}{*}{$\mathrm{HR}^{\mathrm{b}}$} & \multirow{2}{*}{$\begin{array}{c}\text { Flux Density } \\
1 \mathrm{keV}\end{array}$} & \multicolumn{3}{|c|}{ Flux $^{\mathrm{d}}$} \\
\hline & & & & $0.3-8 \mathrm{keV}$ & $0.3-2 \mathrm{keV}$ & $2-8 \mathrm{keV}$ \\
\hline Quasar core & $726 \pm 27$ & $-0.53 \pm 0.03$ & 1.4 & 6.0 & 2.5 & 3.5 \\
\hline Southern hot spot ${ }^{\mathrm{e}}$ & $16.7 \pm 4.1$ & $-0.86_{-0.14}^{+0.04}$ & 0.034 & 0.111 & 0.062 & 0.049 \\
\hline N-C-jet-bend ${ }^{\mathrm{e}}$ & $5.8 \pm 2.5$ & $-0.9 \pm 0.1$ & 0.008 & 0.027 & 0.015 & 0.012 \\
\hline Diffuse emission ${ }^{\mathrm{f}}$ & $22.8 \pm 5.6$ & $-0.09 \pm 0.22$ & 0.05 & 0.13 & 0.09 & 0.04 \\
\hline NGC 4395 B06 & $351 \pm 19$ & $-0.63 \pm 0.04$ & 0.8 & 2.5 & 1.4 & 1.1 \\
\hline
\end{tabular}

Notes.

${ }^{\text {a }}$ Fluxes are determined using the best-fit model in Table 4 unless otherwise noted. Errors are quoted at the $1 \sigma$ level.

${ }^{\mathrm{b}}$ Hardness ratio, $\mathrm{HR}=(H-S) /(H+S)(\mathrm{H}(2-8 \mathrm{keV}), \mathrm{S}(0.3-2 \mathrm{keV}))$, determined using the BEHR method (Park et al. 2006).

${ }^{\mathrm{c}}$ In units of $10^{-13} \mathrm{erg} \mathrm{cm}^{-2} \mathrm{~s}^{-1} \mathrm{keV}^{-1}$.

${ }^{\mathrm{d}}$ In units of $10^{-13} \mathrm{erg} \mathrm{cm}^{-2} \mathrm{~s}^{-1}$; statistical errors in counts dominate for the extended emission.

${ }^{\text {e }}$ Assuming $\Gamma=2$ and Galactic $N_{\mathrm{H}}=1.29 \times 10^{20} \mathrm{~cm}^{-2}$. Southern hot spot includes both SE-hs and pre-hs which are not resolved in the X-ray data.

${ }^{\mathrm{f}}$ Astrophysical Plasma Emission Code model (APEC, http://www.atomdb.org/) for collisionally ionized thermal plasma assuming $k T=4 \mathrm{keV}$; fluxes and flux density are corrected for the $28 \%$ excluded area encompassing the radio-linked X-ray emission.

quasar position $(\alpha=122033.9 ; \delta=+334312)$, and background counts, estimated from an annulus $10^{\prime \prime}-20^{\prime \prime}$ with the same center, were subtracted yielding $726 \pm 27(0.3-8 \mathrm{keV})$ net counts (Table 3). A point-source correction was applied for flux estimates using the CIAO tool arfcorr, which applies an energy-dependent PSF correction appropriate for the extraction region to the ARF (effective area) file. The counts were grouped to yield a minimum of $15 \mathrm{bin}^{-1}$ to perform spectral fitting. A single, absorbed power-law spectral fit in the energy range $0.3-8 \mathrm{keV}$ shows absorption consistent with the Galactic $N_{\mathrm{H}}=1.29 \times 10^{20} \mathrm{~cm}^{-2}$ (Stark et al. 1992), which was accordingly fixed to this value. The resulting best-fit power-law slope, $\Gamma=1.64 \pm 0.08\left(\chi_{\text {red }}^{2} \sim 0.74,42\right.$ degrees of freedom, dof; Figure 3(a)) is consistent with those generally reported for highredshift radio-loud quasars (e.g., Saez et al. 2011; Belsole et al. 2006; Marshall et al. 2005; Cappi et al. 1997). There is an emis- sion line apparent at energy, $E \sim 2.5 \mathrm{keV}$, consistent with cold $\mathrm{Fe} \mathrm{K} \alpha$ in the rest frame of the quasar. Although only marginally significant, addition of a narrow Gaussian line at $2.5 \mathrm{keV}$ improves the fit, while a higher energy line $(2.7 \mathrm{keV}$, approximating ionized $\mathrm{Fe}$ ) is clearly inconsistent. A spectral fit including a narrow line with energy, $E=2.5 \mathrm{keV}$ yields an $\mathrm{EW}=172 \pm 110 \mathrm{eV}$ with a power-law slope $\Gamma=1.66 \pm 0.08\left(\chi_{\text {red }}^{2} \sim 0.70,41 \mathrm{dof}\right)$. Given the large errors, the line energy and equivalent width are consistent with those reported for detected $\mathrm{Fe} \mathrm{K} \alpha$ emission in radio-loud quasars $(\lesssim 100 \mathrm{eV}$, Grandi et al. 2006). There is a suggestion (Figure 3(b)) of further emission lines around 0.8 and $0.5 \mathrm{keV}$ which roughly align with highly ionized $\mathrm{Si}$ and $\mathrm{Mg}$ features in a photoionized spectrum in the quasar rest frame. However, they are not significant in this low-count spectrum. The fit parameters with and without the $\mathrm{Fe} \mathrm{K} \alpha$ emission line are given in Table 4. Consistency of the data with the Galactic $N_{\mathrm{H}}$ 
Table 4

Parameters for X-Ray (0.3-8 keV) Spectral Fits ${ }^{\mathrm{a}}$

\begin{tabular}{lcccccc}
\hline \hline Region Name & Model & $\Gamma$ & $\begin{array}{c}\text { Line E } \\
(\mathrm{keV})\end{array}$ & $\begin{array}{c}\text { Line EW }^{\mathrm{b}} \\
(\mathrm{eV})\end{array}$ & $\chi^{2}$ & dof $^{\mathrm{c}}$ \\
\hline Quasar core $^{\mathrm{d}}$ & PL+Gauss & $1.66 \pm 0.08$ & 2.5 & $172 \pm 110$ & 28.7 & 41 \\
& PL & $1.68 \pm 0.08$ & $\ldots$ & $\ldots$ & 31.3 & 42 \\
NGC 4395 B06 & PL & $1.99 \pm 0.15$ & $\ldots$ & $\ldots$ & 29.4 & 21 \\
\hline
\end{tabular}

Notes.

a Assuming Galactic $N_{\mathrm{H}}=1.29 \times 10^{-20} \mathrm{~cm}^{-2}$. Errors are quoted at the $1 \sigma$ level for one interesting parameter (unless otherwise noted).

b Equivalent width of emission line.

${ }^{c}$ Data were re-binned with a minimum of 15 counts bin ${ }^{-1}$.

${ }^{\mathrm{d}}$ Errors are $1 \sigma$ for two interesting parameters.

indicates no evidence for intrinsic absorption or the presence of a soft excess component, nor is there any improvement to the fit if a reflection component is added. Both intrinsic absorption and reflection components tend to be weak in face-on, type I quasars, particularly those that are radio loud such as 3C 270.1 (Molina et al. 2008; Saez et al. 2011).

\subsubsection{Radio-Lobe-associated X-Ray Emission}

$\mathrm{X}$-ray emission is present coincident with the southern radio lobe, and the peak is close to the primary (SE) radio hot spot. The net counts were extracted from a circle with a $1^{\prime \prime}$.7 radius centered on $\alpha=122033.91, \delta=+3343$ 07.25, which includes the cluster of counts in this region and aligns well with the position of the double radio hot spot. The net counts, $16.7 \pm$ 4.1 , are primarily soft, with a hardness ratio $\mathrm{HR}=-0.86_{-0.14}^{+0.04}$. There are too few counts for a spectral fit. Fluxes (Table 3) are insensitive to the assumed spectral form since statistical uncertainties dominate.

$\mathrm{X}$-ray emission north of the quasar is centered SE of the radio hot spot in the northern radio lobe, aligned with the position of the N-C-jet-bend in the radio emission (Figure 1). Counts were extracted from a circular region, radius $1^{\prime \prime} .25$, centered on $\alpha=12: 20: 33.74, \delta=33: 43: 15.4$, selecting the cluster of events apparently associated with the radio emission in this region of sky. We find six counts where 0.2 background counts are expected, a significant detection. The net counts, $5.8 \pm 2.5$, in the broadband $(0.3-8 \mathrm{keV})$ are soft with all the counts below $2 \mathrm{keV}$ giving a hardness ratio, $\mathrm{HR}=-0.9 \pm 0.1$ (Table 3 ).

There is no detected X-ray emission associated with the northern radio hot spot. The more extended lobe emission possibly includes faint X-ray emission at its southern edge, but the association is highly uncertain given the low signal-to-noise ratio $(\mathrm{S} / \mathrm{N})$ and the lack of alignment with the lobe.

\subsubsection{Nearby X-Ray Source: NGC 4395 B06}

There is a strong point-like X-ray source $48^{\prime \prime}$ off-axis, close enough that there is no significant change in the PSF, on the same ACIS S-3 chip, and located at the position of the radioquiet quasar NGC 4395 B06 $(\alpha=122032.7, \delta=+334356$, $z=1.038$; Barkhouse \& Hall 2001). Extracting counts from a circular region with a $1^{\prime \prime} .5$ radius, as for $3 \mathrm{C} 270.1$, and estimating background counts from a $5^{\prime \prime}-10^{\prime \prime}$ annulus centered on the quasar yields $351 \pm 19$ net counts. The hardness ratio, $\mathrm{HR}=-0.63 \pm 0.04$, is similar to that of the nucleus of 3C 270.1 (Table 3). A power-law fit assuming Galactic $N_{\mathrm{H}}$ yields $\Gamma=1.99 \pm 0.15\left(\chi_{\text {red }}^{2}=0.89,21\right.$ dof; Figure 4, Table 4). Fitting with $N_{\mathrm{H}}$ free results in a slightly flatter power-law slope

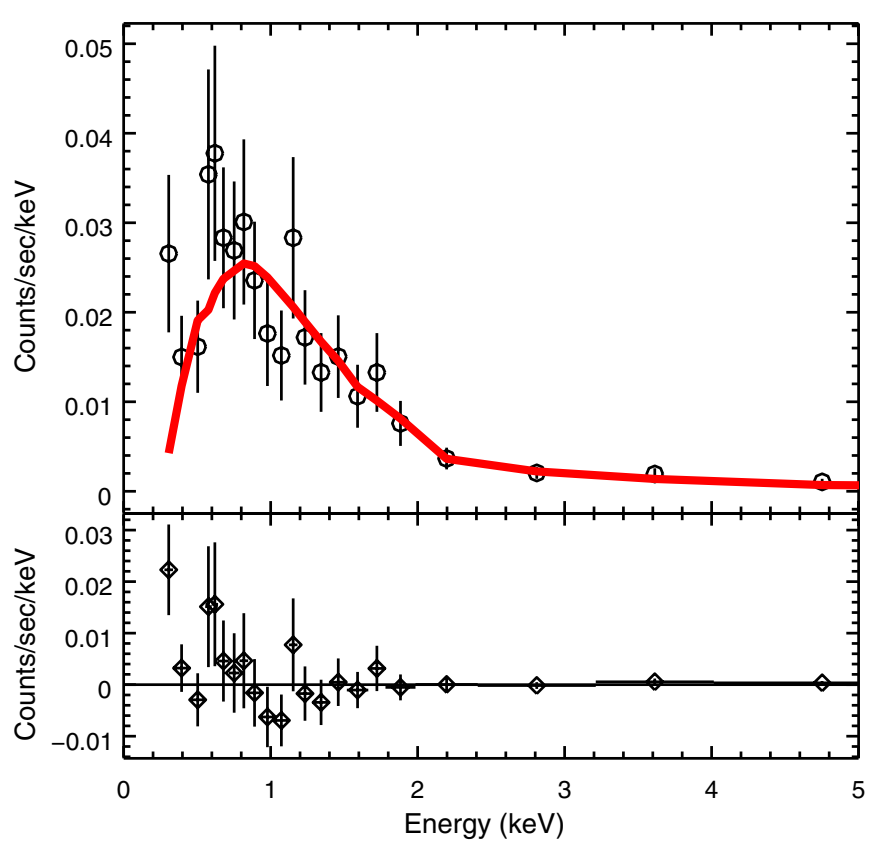

Figure 4. Best-fit single power-law $\left(\Gamma=1.99 \pm 0.15\right.$, Galactic $\left.N_{\mathrm{H}}\right)$ spectral model for NGC4395 B06 superposed on the X-ray data with the residuals below. (A color version of this figure is available in the online journal.)

$\left(\Gamma=1.92 \pm 0.14, \chi_{\text {red }}^{2}=0.87,20\right.$ dof $)$ and very low $N_{\mathrm{H}}$, suggesting the absorption is offset by a soft excess component. However the $\mathrm{S} / \mathrm{N}$ is insufficient to distinguish between models for a soft excess and the power-law slope is typical of radio-quiet quasars (Piconcelli et al. 2005) so no more complex modeling was carried out. Fluxes were determined using the initial fit, assuming Galactic $N_{\mathrm{H}}$.

\subsubsection{Diffuse Extended Emission around 3C 270.1}

There are additional X-ray counts around the quasar core which are not associated with the envelopes of radio emission. The emission occurs on both sides of the quasar but the number of counts is too low to constrain its spatial distribution.

We extracted counts in the broadband $(0.3-8 \mathrm{keV})$ from an annular region centered on $3 \mathrm{C} 270.1$ and extending from $2^{\prime \prime}$ to $7^{\prime \prime} .5$ ( $\sim 64 \mathrm{kpc}$ ) excluding two segments (opening angles: north: $60^{\circ}$, south: $40^{\circ}$ ) containing the radio-related regions; this excluded $28 \%$ of the annulus. Background counts (34) were estimated from an annular region extending from $10^{\prime \prime}$ to $20^{\prime \prime}$. Twenty-seven counts were detected where $4.3 \pm 0.7$ are expected, a detection which is highly significant (Table 3 ). The net extended counts are $22.8 \pm 5.6(0.3-8 \mathrm{keV})$ with an energy distribution harder $(\mathrm{HR}=-0.09 \pm 0.22)$ than the quasar or the radio-linked X-rays. Extracting counts from a similar extended region $(2$ ".5-7".5) around the bright, nearby quasar NGC4395 B06 yields only 9 counts where $5 \pm 1$ are expected, a marginal detection of 3.9 counts. We thus conclude that $<20 \%$ of the diffuse extended counts around 3C 270.1 could originate in the wings of the central quasar. Since the wings are more extended at soft energies, this would result in an even harder spectrum for the diffuse emission.

The hardness of the diffuse counts makes it unlikely that they are nuclear X-rays Thomson-scattered from an extended gaseous halo. They could be CMB photons Compton-scattered by electrons associated with fainter, more diffuse radio emission below the current radio flux limit, as suggested for the high- 

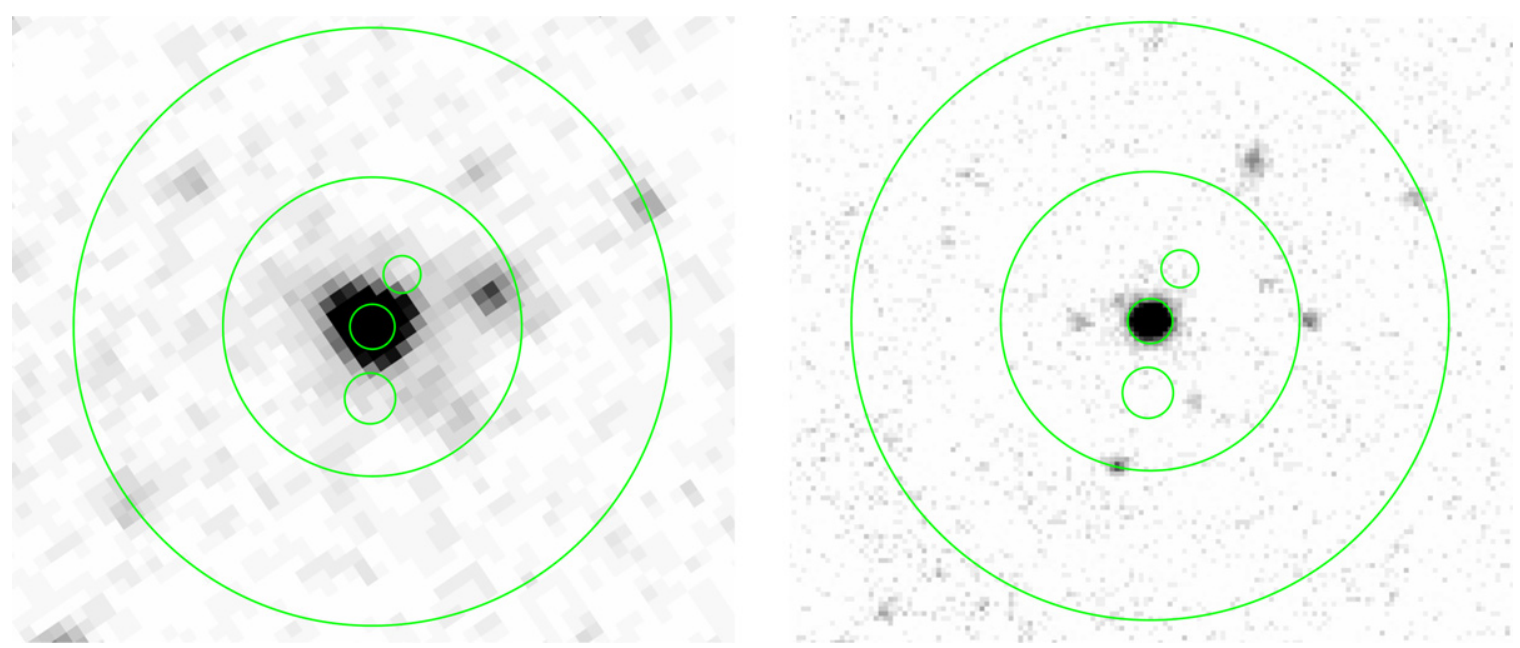

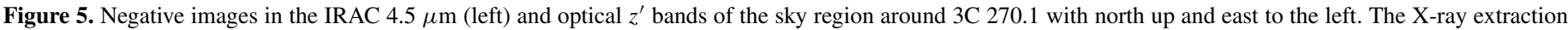

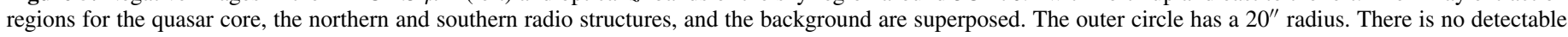
IR or optical emission associated with the radio lobes.

(A color version of this figure is available in the online journal.)

redshift radio source 3C 294 (Fabian et al. 2003), or thermal emission from hot gas in the optical/IR cluster (Haas et al. 2009).

Since the mean surface brightness of the diffuse emission is 0.18 counts $\operatorname{arcsec}^{-2}$, it might contribute 1.6 and 0.9 counts in the extraction regions for the southern hot spot and northern $\mathrm{N}-\mathrm{C}$-jet-bend, $6 \%$ and $15 \%$ of the detected counts respectively, assuming it is isotropic. Correction of the X-ray spectra of the radio features would result in even softer emission and somewhat lower fluxes in these regions (Table 3). Any contribution to the quasar core would be negligible $(\sim 1.3$ of the 726 net counts).

\subsection{Spitzer and Optical $z^{\prime}$ Data Analysis}

The $4.5 \mu \mathrm{m}$ IRAC data for 3C 270.1 are shown in Figure 5 with the $\mathrm{X}$-ray extraction regions superposed. The quasar is well detected in the IRAC data. There is no detected emission associated with either of the radio lobes. Upper limits were determined for the radio hot spots and the $\mathrm{N}$-C-jet-bend appropriate for a point source since any associated IR emission would be unresolved with Spitzer. A second set of upper limits, appropriate for extended IR emission features, was determined for the full southern radio lobe based upon the radio contours. The upper limits are given in Table 2 and used to constrain the spectral energy distribution (SED) models described in Section 4.

The $z^{\prime}$ optical image is also shown in Figure 5 with the X-ray extraction regions superposed. There is no detectable optical emission at the position of the radio lobes. Upper limits were determined appropriate to the X-ray regions. We estimated the sensitivity from the fluctuations measured in source-free pixels to be $21.8 \mathrm{AB}$ mag $(6.9 \mu \mathrm{Jy}, 5 \sigma)$ within a $3^{\prime \prime}$ diameter aperture.

There is a faint source $\sim 6^{\prime \prime}$ east of $3 \mathrm{C} 270.1$, also marginally visible in the IRAC data. It is most likely a faint galaxy. The position is $\sim 1$ ". 4 east of a small group of X-ray photons currently identified as part of the extended, diffuse emission. Given this offset, the X-ray counts are unlikely to be related, but deeper Chandra X-ray data would clarify any possible association.

\section{MODELING THE X-RAY EMISSION FROM HOT SPOTS AND RADIO LOBES}

There are three mechanisms commonly invoked to explain radio-associated, extended X-ray emission: synchrotron, SSC, and $\mathrm{iC} / \mathrm{CMB}$. All three were applied to the components of 3C 270.1 following the method described by Hardcastle et al. (1998). Given the lower spatial resolution and low $\mathrm{S} / \mathrm{N}$ of the Chandra data, we are unable to uniquely identify the $\mathrm{X}$-ray emission with specific radio structure based on spatial distribution alone. We therefore determined the fluxes from the north and south radio structures in regions appropriate for the observed X-ray emission and constructed radio through X-ray SEDs for the radio regions assuming, in each case, that all the $\mathrm{X}$-ray emission is associated with the particular radio region being considered. The models were then applied to each SED.

The radio through X-ray SED for each region was fitted with a standard continuous injection electron spectrum with a minimum energy corresponding to $5 \times 10^{6} \mathrm{eV}$ and maximum energy corresponding to $5 \times 10^{12} \mathrm{eV}$ for the S-full and N-Cjet-bend. For the SE-hs, we used the identical minimum energy but a maximum energy of $5 \times 10^{10} \mathrm{eV}$. The choice of highenergy cutoff was due to the following two constraints: (1) it is as low as possible without being inconsistent with the radio data, and (2) the fitted synchrotron model of these data does not violate the Spitzer upper limits. There remain significant uncertainties in the maximum electron energy, but for $\alpha>0.5$ and $E_{\max } \gg E_{\min }$ the derived physical parameters are not very sensitive to this value. We assume that non-radiating particles are insignificant in the radio plasma and use a filling factor of 1.

The predicted X-ray flux density for each region was determined for a broken power-law synchrotron model with a high-energy cutoff, a synchrotron self-Compton model (SSC) in which the X-ray emission is the result of $\mathrm{iC}$ scattering of the synchrotron radio photons off the relativistic electrons, and a model of $\mathrm{iC}$ scattering of $\mathrm{CMB}$ photons (iC/CMB) off the radio synchrotron-emitting relativistic electrons, respectively. The models and predictions were determined in the quasar's rest frame and are shown in Figures 6 and 7 superposed on the observed SED. The infrared upper limits (at 3.6 $\sigma$ ) for each 

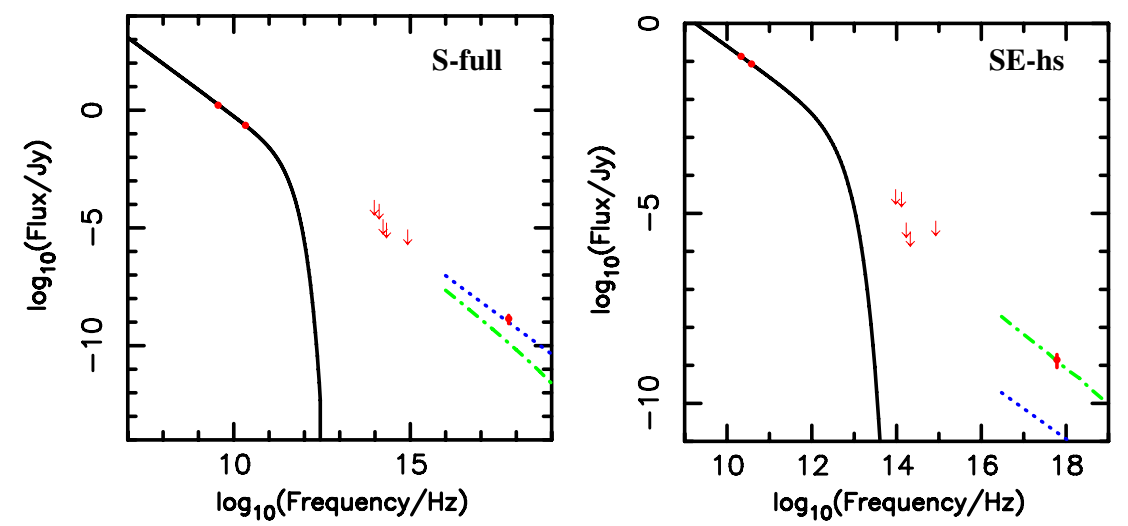

Figure 6. Left: spectral energy distribution (SED) for the southern radio lobe (S-full, Table 2). The synchrotron model consistent with the radio data (black line) and the predicted X-ray emission from SSC (green dash-dotted line) and iC/CMB (blue dotted line) for a magnetic field of $8 \mathrm{nT}$ are superposed. The model is based on the VLA data for the lobe with the flux density of the hot spot subtracted. Right: the SED for the SE component of the double hot spot (SE-hs in Table 2) with the synchrotron model and predicted X-ray emission levels superposed. SSC emission with a field of $24 \mathrm{nT}$ (roughly a third of the equipartition field) is consistent with the data. In both figures the data and model are blueshifted to the source frame, the red data points show the radio and X-ray flux densities and red arrows show the Spitzer and $z^{\prime}$ upper limits. The error bars indicate $1 \sigma$ statistical uncertainties, which are smaller than the radio data points.

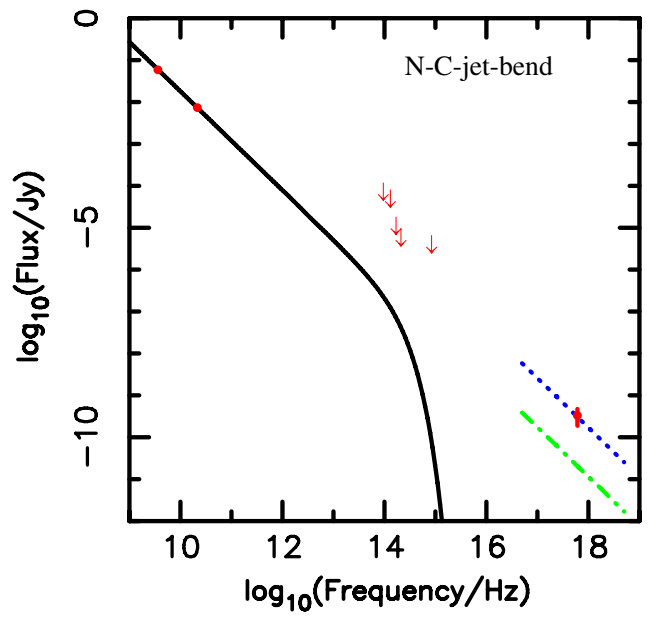

Figure 7. SED for the N-C-jet-bend region, on the east side of the northern radio lobe (Table 2 ) with the direct synchrotron component matched to the VLA data (black) and the predicted SSC (green) and iC/CMB (blue) X-ray emission adopting a magnetic field of $3 \pm 1 \mathrm{nT}$, a factor of 7-10 lower than the equipartition field. The data and model are blueshifted to the source frame, symbols are as in Figure 6.

region from the Spitzer IRAC data sometimes constrain the highest energy electron populations. The figures also include a $z^{\prime}$ upper limit appropriate for a point source, but this does not provide an independent constraint.

\subsection{Southern Radio Lobe/Hot Spot}

The $\mathrm{S} / \mathrm{N}$ of the Chandra data is too low for us to determine whether the X-ray emission arises from the entire southern radio lobe or the hot $\operatorname{spot}(\mathrm{s})$. If the $\mathrm{X}$-ray emission originates throughout the lobe, the synchrotron and SSC predictions are 1-2 orders of magnitude below the observed X-ray flux while the $\mathrm{iC} / \mathrm{CMB}$ prediction is consistent with the observed X-ray flux if the magnetic field is set at $8 \mathrm{nT}$, about a quarter of the equipartition field for this region (Figure 6, left).

If instead all the X-ray emission originates in the SE hot spot, as suggested by the alignment of the peak emission, the Spitzer and optical upper limits rule out direct synchrotron as a viable mechanism for the emission at IRAC and shorter wavelengths, including the X-ray emission. The
$\mathrm{iC} / \mathrm{CMB}$ prediction for this small region is $\sim 3$ orders of magnitude too low. The SSC prediction with a minimum-energy equipartition magnetic field of $86 \mathrm{nT}$ is also low but can be brought into agreement with a field of $24 \mathrm{nT}$ (Figure 6, right), about a third of this value. This is not an unusual departure (Hardcastle et al. 2004) and not surprising given the uncertainties involved. For example, the X-ray emission could originate in more than one component and allow for a model closer to the equipartition field.

The radio emission from the precursor hot spot region (SE-pre-hs, Table 2) can only be determined at one frequency. Given the lower spatial resolution of the Chandra data and the lack of constraint on the optically thin part of the radio spectrum and subsequent extrapolation of the electron spectrum to low energies, we cannot rule out a significant contribution from SSC emission from the precursor region.

In summary, the most likely interpretation, given the alignment between the X-ray emission and the radio emission, is that the X-rays primarily originate in the hot spot and/or the precursor hot spot and are due to SSC. For emission only from the SE hot spot, the field is $\sim 24 \mathrm{nT}$, about a third of the equipartition field.

\subsection{Northern Radio Emission}

There is no detectable X-ray emission aligned with the northern hot spot. The SSC X-ray prediction for the northern hot spot, for an equipartition field of $77 \mathrm{nT}$ based on the radio flux, assuming $\alpha=0.7$ and constrained by the IR upper limits, is about a factor of 10 lower than the X-ray upper limit $\left(1.3 \times 10^{-4} \mu \mathrm{Jy}, 3 \sigma\right)$ so that no X-ray emission is expected.

There is significant X-ray emission aligned with the $\mathrm{N}-\mathrm{C}$-jetbend (Table 2), although with only a few counts the uncertainty on the flux is high. Assuming the radio plasma is static, the SSC predictions for an equipartition magnetic field of $28 \mathrm{nT}$ are a factor $\geqslant 10^{2}$ lower than the X-ray flux density. The iC/CMB prediction is closer and can be brought into agreement with the observations if the magnetic field is $3 \pm 1 \mathrm{nT}$ (Figure 7). This field is a factor of $\sim 7-10$ lower than equipartition, a larger departure than is generally observed (Croston et al. 2005). Since we do not have observations of radio emission from the lowenergy electrons responsible for the $\mathrm{iC} / \mathrm{CMB} \mathrm{X}$-rays, that radioemitting region may be more extended (as, e.g., in Schwartz et al. 
2006), which would result in a field closer to the equipartition value. Our upcoming deeper Chandra observations (approved in Cycle 13) will provide improved constraints on these models.

\section{DIFFUSE, EXTENDED X-RAY EMISSION UNASSOCIATED WITH RADIO STRUCTURES}

There is evidence for a cluster surrounding 3C 270.1 in optical and infrared data (Haas et al. 2009). An obvious question, therefore, is whether the diffuse X-rays which are not associated with the radio structures could be thermal emission from $\mathrm{X}$-ray gas in the cluster. For the X-rays to be cluster emission, we would expect a smooth and relatively isotropic distribution. The cluster center is not well constrained. It was estimated to lie $\sim 20^{\prime \prime}$ east of 3C 270.1 by Haas et al. (2009). The current data set has insufficient observed counts $(22.8 \pm 5.6,0.3-8 \mathrm{keV})$ to constrain the spatial distribution of the diffuse emission. However, the existence of an excess of counts around 3C 270.1 and the lack of a similar excess to the east suggest that the quasar may lie close to the center of the cluster.

The extended emission has too few counts to constrain the spectral parameters. The hardness ratio $\mathrm{HR}=-0.09 \pm 0.22$ indicates a temperature of $\sim 4 \mathrm{keV}$ in an APEC model but remains within $2 \sigma$ of a power-law spectrum with $\Gamma \sim 1.7$, so we cannot rule out non-thermal emission. An APEC model was fitted, assuming abundance $Z=0.5 Z_{\odot}$ and temperature $4 \mathrm{keV}$ at the redshift of the source. With a grouping of 5 counts bin ${ }^{-1}$ to distribute them throughout the band, the spectrum looks reasonable, but the $\mathrm{S} / \mathrm{N}$ is too low to provide meaningful constraints. The fitted normalization yields a broadband flux, $\mathrm{F}(0.3-8 \mathrm{keV})=(1.1 \pm 0.5) \times 10^{-14} \mathrm{erg} \mathrm{cm}^{-2} \mathrm{~s}^{-1}$, including a correction for the excluded sky area assuming isotropic emission. This translates to a broadband X-ray luminosity $\sim 2 \times 10^{44} \mathrm{erg} \mathrm{s}^{-1}$ for our assumed cosmology, consistent with the luminosity of a $4 \mathrm{keV}$ cluster based on the low-redshift luminosity-temperature $\left(L-T,<R_{500}\right)$ relation of Pratt et al. (2009). The few high-redshift $(z>1.4)$ clusters with X-ray measurements tend to be faint for their temperature based on a self-similar model for their evolution in comparison with similarly selected lower-redshift clusters (Andreon et al. 2011; Hilton et al. 2010). Our uncertainties are too large to test this for 3C 270.1. Early cluster formation, consistent with a cluster around 3C 270.1, would be challenging for cluster formation models, which suggest that massive dark matter halos form primarily at $z \lesssim 1.2$ (Croton et al. 2007).

The data are too limited to constrain the X-ray spatial distribution, temperature, or abundances of the diffuse component. Our analysis is based on $\sim 60 \mathrm{kpc}$ close to or at the center of the cluster, where the physical conditions and the relation to the full $R_{500}$ luminosity and temperature depend on the presence/absence of a cooling core (Pratt et al. 2009) and can be disturbed by heat input by the quasar. Deeper Chandra Xray observations are required to confirm the extended nature and constrain the spatial distribution, spectral properties, and luminosity of the diffuse X-ray emission.

Qualitatively, the observations of extended X-ray emission are consistent with the asymmetric depolarization of the radio source observed by Garrington et al. (1991; see Section 3.1). For a more quantitative comparison we can consider the simple analysis of Garrington \& Conway (1991), which assumes that the density profile of the cluster can be modeled as a $\beta$ model and that the energy density in the magnetic field of the depolarizing medium scales as the energy density in thermal particles. The observed ratio of the dispersions in the Faraday depth in the two lobes, $r_{\Delta}$ in the notation of Garrington \& Conway, is $\sim 2$. Since we detect an excess of counts close to the quasar, the core radius $a$ of any $\beta$ model that represents the observed $\mathrm{X}$-ray emission must be comparable to or less than the size of our extraction region, with an outer radius of $7^{\prime \prime} .5(64 \mathrm{kpc})$. From the radio data, the projected linear size of each lobe is $6^{\prime \prime}(\sim 50 \mathrm{kpc})$. The combination of these values and the observed $r_{\Delta}=2$ suggest that the gas distribution is rather flat $(\beta \lesssim 0.35)$ and that the lobes are not aligned close to the line of sight. However, the current quality of X-ray data on the gas near 3C 270.1 is too low to provide useful constraints. Our upcoming deeper Chandra observations of this source, or Chandra observations of a larger sample of objects with observed depolarization, will give us a probe of the run of gas density and magnetic field strength with radius in high- $z$ clusters which will be difficult to obtain in any other way.

\section{CONCLUSIONS}

Chandra X-ray observations of the bright, high-redshift $(z=1.532)$ quasar 3C 270.1 show strong, unabsorbed powerlaw emission with a slope $\Gamma=1.66 \pm 0.08$, consistent with expectations for radio-loud quasars.

Extended X-ray emission associated with the southern radio lobe of 3C 270.1 most likely originates in one/both components of the double hot spot within that lobe. Spitzer upper limits for the hot spot are inconsistent with synchrotron emission from a single power-law population of electrons as the emission mechanism. The X-ray emission is consistent with SSC for a magnetic field of $24 \mathrm{nT}$, about a third of the equipartition field for that region.

No X-ray emission is detected from the northern radio hot spot as expected from our models based on the observed SED. Faint emission is present just south of the lobe but seems unlikely to be associated given the lack of alignment.

Weak but significant X-ray emission associated with the southeastern extension of the northern radio-lobe, coincident with a bend in the radio counter-jet ("N-C-jet-bend"), can be explained by $\mathrm{iC} / \mathrm{CMB}$ emission with a field of $3 \pm 1 \mathrm{nT}$, a factor of 7-10 lower than the equipartition field. This is a larger departure than is typical suggesting that, for example, the emitting region may be larger than our estimate.

Extended X-ray emission unassociated with the observed radio structures may be thermal emission from gas within the cluster believed to be surrounding the quasar or iC emission associated with radio emission below the present detection limit. There are too few counts to constrain its spatial distribution or spectrum, but assuming a temperature of $4 \mathrm{keV}$ yields a luminosity $\sim 2 \times 10^{44} \mathrm{erg} \mathrm{s}^{-1}$, consistent with the low-redshift $L-T$ relation and suggestive of a fully formed cluster at redshift 1.532 .

Upcoming deeper Chandra and radio (Expanded Very Large Array) observations will allow us to confirm the location and origin of the radio-associated X-ray emission for both northern and southern radio emission structures, to study the spatial distribution of the diffuse $\mathrm{X}$-ray emission and its relation to any fainter, diffuse radio emission or to the optical/IR cluster, and to constrain its spectral form.

Support for this work was provided by the National Aeronautics and Space Administration through Chandra Award Number G08-9106X issued by the Chandra X-ray Center, which is operated by the Smithsonian Astrophysical Observatory for and on behalf of the National Aeronautics Space Administration 
under contract NAS8-03060 (Chandra X-ray Center). The National Radio Astronomy Observatory is a facility of the National Science Foundation operated under cooperative agreement by Associated Universities, Inc. Observations reported here were obtained at the MMT Observatory, a joint facility of the Smithsonian Institution and the University of Arizona. This work is based in part on observations made with the Spitzer Space Telescope, which is operated by the Jet Propulsion Laboratory, California Institute of Technology, under a contract with NASA.

\section{REFERENCES}

Andreon, S., Trinchieri, G., \& Pizzolato, F. 2011, MNRAS, 412, 2391 Barkhouse, W. A., \& Hall, P. B. 2001, AJ, 121, 2843

Belsole, E., Worrall, D. M., \& Hardcastle, M. J. 2006, MNRAS, 366, 339

Cappi, M., Matsuoka, M., Comastri, A., et al. 1997, ApJ, 478, 492

Croston, J. H., Hardcastle, M. J., Harris, D. E., et al. 2005, ApJ, 626, 733

Croton, D. J., Gao, L., \& White, S. D. M. 2007, MNRAS, 374, 1303

Fabian, A. C., Sanders, J. S., Crawford, C. S., \& Ettori, S. 2003, MNRAS, 341, 729

Fanaroff, B. L., \& Riley, J. M. 1974, MNRAS, 167, 31P

Fukugita, M., Ichikawa, T., Gunn, J. E., et al. 1996, AJ, 111, 1748
Garrington, S. T., \& Conway, R. G. 1991, MNRAS, 250, 198

Garrington, S. T., Conway, R. G., \& Leahy, J. P. 1991, MNRAS, 250, 171 Grandi, P., Malaguti, G., \& Fiocchi, M. 2006, ApJ, 642, 113

Haas, M., Willner, S. P., Heymann, F., et al. 2008, ApJ, 688, 122

Haas, M., Willner, S. P., Heymann, F., et al. 2009, ApJ, 695, 724

Hardcastle, M. J., Birkinshaw, M., \& Worrall, D. M. 1998, MNRAS, 294, 615

Hardcastle, M. J., Harris, D. E., Worrall, D. M., \& Birkinshaw, M. 2004, ApJ, 612,729

Harris, D. E., \& Krawczynski, H. 2002, ApJ, 565, 244

Hilton, M., Lloyd-Davies, E., Stanford, S. A., et al. 2010, ApJ, 718, 133

Larson, D., Dunkley, J., Hinshaw, G., et al. 2011, ApJS, 192, 16

Lonsdale, C. J., \& Barthel, P. D. 1984, A\&A, 135, 45

Lonsdale, C. J., Barthel, P. D., \& Miley, G. K. 1993, ApJS, 87, 63

Marshall, H. L., Schwartz, D. A., Lovell, J. E. J., et al. 2005, ApJS, 156, 13

McLeod, B., Geary, J., Ordway, M., et al. 2006, in Scientific Detectors for Astronomy 2005, ed. J. E. Beletic, J. W. Beletic, \& P. Amico (Berlin: Springer), 337

Molina, M., Bassani, L., Malizia, A., et al. 2008, MNRAS, 390, 1217

Park, T., Kashyap, V. L., Siemiginowska, A., et al. 2006, ApJ, 652, 610

Piconcelli, E., Jimenez-Bailón, E., Guainazzi, M., et al. 2005, A\&A, 432, 15

Pratt, G. W., Croston, J. H., Arnaud, M., \& Böhringer, H. 2009, A\&A, 498, 361 Saez, C., Brandt, W. N., Shemmer, O., et al. 2011, ApJ, 738, 53

Schwartz, D. A., Marshall, H. L., Lovell, J. E. J., et al. 2006, ApJ, 647, L107

Stark, A. A., Gammie, C. F., Wilson, R. W., et al. 1992, ApJS, 79, 77

Venemans, B. P., Röttgering, H. J. A., Miley, G. K., et al. 2007, A\&A, 461, 823 Worrall, D. M. 2009, A\&AR, 17, 1 